\title{
Special Cluster on Antennas and Propagation Technologies in Conjunction with Main Topics of ISAP2020
}

The 2020 International Symposium on Antennas and Propagation (ISAP2020), the 25th ISAP, sponsored by the Communications Society of the IEICE, held at Knowledge Capital Congrès Convention Center in Osaka, Japan on January 25 to 28,2021 was a great success. This special cluster includes submissions particularly from, but not limited to, the authors of ISAP2020.

Since review decision of the IEICE Communications Express (ComEX) has no conditional acceptance, this cluster provided two submission deadlines to make a chance for revised submission. For this cluster, 20 and 23 letters were submitted by the first and the second deadlines. After careful review, 19 letters were accepted in total.

This is the 5th special cluster in ComEx, and the 3rd one on antennas and propagation technologies. I am grateful to the Editorial Committee of ComEX for deciding to publish this special cluster and their continuous support. And, I would like to express my sincere appreciation to all authors for their excellent contributions, and to the reviewers and editorial committee members for their great effort to make this special cluster successful.

Finally, I sincerely hope that the Special Cluster Series on Antennas and Propagation Technologies will encourage researchers and will promote further research activities in the related fields of study.

\section{Guest Editor-in-Chief:}

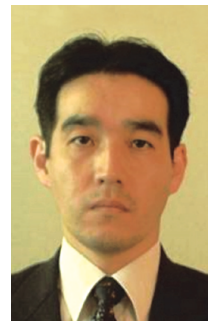

Ryo Yamaguchi (SoftBank)

\section{Special Cluster Editorial Committee Members}

Guest Editors: Kentaro Saito (Tokyo Denki Univ.), Takuji Arima (Tokyo Univ. of Agriculture and Tech.)

Guest Associate Editors: Tetsuro Imai (Tokyo Denki Univ.), Kunio Sakakibara (Nagoya Inst. of Tech.), Keisuke Noguchi (Kanazawa Inst. of Tech.), Takuya Sakamoto (Kyoto Univ.), Shinichiro Ohnuki (Nihon Univ.), Kazunari Kihira (Mitsubishi Electric), Mitoshi Fujimoto (Univ. of Fukui), Eisuke Nishiyama (Saga Univ.), and ComEX Associate Editors 